\section{A response to Pellicano et al.}

\section{Pat Walsh, Mayada Elsabbagh, Patrick Bolton and Ilina Singh}

In our examination of the scientific, social and ethical implications of research into autism biomarkers we called for a widespread debate involving many diverse parties and including, importantly, scientists and members of the autism community and their carers (In search of biomarkers for autism: scientific, social and ethical challenges. Nature Rev. Neurosci. 12, 603-612 (2011)) ${ }^{1}$. We are pleased that Pellicano et al. (Engaging, not excluding: a response to Walsh et al. Nature Rev. Neurosci. 18 Nov 2011) ${ }^{2}$ reach the same conclusion in their commentary on our Perspective article. However, they claim that what we say is "confused" and, puzzlingly, that the involvement of members of the autism community and their carers in the biomarker research process which we strongly advocate - would be precluded by our recommendations. They do not state precisely where the confusion lies nor do they clarify which recommendations they have in mind. We are left to suppose that what they object to is the research programme itself because of its possible implications for reproductive decision-making - and our (nuanced) acceptance of it. Our article ${ }^{1}$ outlined the large scientific challenges that biomarker research has yet to overcome and also addressed the main social and ethical issues - and risks to which its eventual successes could give rise. Our article did assume, nevertheless, that the search for biomarkers for autism will continue, given the depth and range of their potential benefits for people with autism and their families. Special emphasis was placed on the importance of the distinction between rightful acceptance and rightful intervention regarding autism in the context of prevention. We also acknowledged the moral gravity of some possible consequences of biomarker knowledge for reproductive decision-making. Pellicano et al. ${ }^{2}$ object to our emphasis on fully informed parental decision-making, which they suggest begs the question of who should make the final decision. In response, one is tempted to ask how it could be otherwise in an era of reproductive freedom, but what we said in our article was only that legal permission would not diminish the moral seriousness of parental choices. Given this seriousness, the structured public consideration of these ethical issues that we called for in our article, far from precluding members of the autism community, needs their full participation.

A second objection made is that our article does not query the disproportionate funding of aetiological research as opposed to other areas of research. It is true that, although we indicate concern about the proper ends of research, we do not address the current flow of research resources. However, given the promise of aetiological research and the only modest benefits that are currently achieved by intervention research, it is not obvious that clinical and translational research has, in principle, more immediate applications than biomedical research. But, in any case, our focus on biomarkers was precisely against the background of their translational potential as demonstrated in other areas of medicine. Furthermore, one of our key conclusions about autism was that the challenges that biomedical research needs to surmount include heterogeneity, a complex clinical phenotype, and a lack of agreed and stable definitions of autism - the very obstacles that also hinder evidence-based practice in the clinical and translational research community. It is reasonable to suppose that an improved understanding of the pathways that lead to autism, and of how it alters neurobiological functioning, is most likely to lead to interventions that will positively affect the experience of individuals and families that are affected by the condition. However, we are by no means opposed to the monitoring of funding streams or to improved funding for other forms of research (noting, too, that gaining and distributing funding does not have to be a zero-sum game). We also acknowledge the great importance of concerns about the quality of life of people affected by autism now, and about how best to address these problems. The balancing acts required in setting research policy further underline the need for the collaborative approach that we advocate in our article.

Pat Walsh is at the Centre of Medical Law and Ethics, School of Law, Kings College London, London WC2R 2LS, UK.

Mayada Elsabbagh is at the Department of Psychiatry, McGill University, 1033 Pine Avenue West, Montreal, Quebec H3A 1A1, Canada.

Patrick Bolton is at the Institute of Psychiatry, Kings College London, De Crespigny Park, London SE5 8AF, UK

Ilina Singh is at the BIOS Centre, London School of Economics and Political Science, London WC2A $2 A E$, UK

Correspondence to P.W. and M.E. e-mails: patricia.walsh@kcl.ac.uk; mayada.elsabbagh@mcgill.ca doi:10.1038/nrn3113-c2

1. Walsh, P., Elsabbagh, M., Bolton, P. \& Singh, I. In search of biomarkers for autism: scientific, social and ethical challenges. Nature Rev. Neurosci. 12 , 603-612 (2011).

2. Pellicano, E., Ne'eman, A. \& Stears, M. Engaging, not excluding: a response to Walsh et al. Nature Rev. Neurosci. 18 Nov 2011 (doi: 10.1038/nrn3113-c1).

Competing interests statement

The authors declare no competing financial interests. 